\title{
Territoires d'élevage pastoral au Sahel : un bilan carbone avec un potentiel inattendu d'atténuation du changement climatique
}

\author{
Mohamed Habibou Assouma - Philippe Lecomte - Christian Corniaux - \\ Pierre Hiernaux - Alexandre Ickowicz - Jonathan Vayssières
}

\begin{abstract}
Au Sahel, l'élevage pastoral valorise un milieu extrême. Alors qu'il est accusé d'émettre trop de gaz à effet de serre par kilogramme de lait ou de viande produit, une recherche menée au Sénégal montre que les territoires pastoraux peuvent en réalité avoir un bilan carbone neutre : les émissions d'origine animale sont compensées par la séquestration de carbone dans les sols et la végétation. Ces résultats ont été obtenus par une méthode d'évaluation originale, dite écosystémique : elle intègre l'utilisation du territoire pastoral dans son ensemble, en fonction
\end{abstract}

/ élevage pastoral est présent dans toutes les régions du monde et il fait vivre plus de 100 millions de personnes. II concerne surtout les ruminants - bovins, ovins, caprins, camélidés, cervidés. Dans ce type d'élevage extensif, les troupeaux parcourent les territoires pour se nourrir de la végétation et pour s'abreuver. En Afrique, le pastoralisme représente 10 à $44 \%$ du produit intérieur brut selon les pays et il occupe plus d'un quart de leur population. Dans les pays du Sahel, il fournit $70 \%$ du lait et plus de la moitié de la viande de bovins et de petits ruminants.

Cependant, à l'échelle mondiale, le secteur de l'élevage contribue à $14,5 \%$ des émissions de gaz à effet de serre liées aux activités humaines, selon la Fao - les émissions dues à l'élevage étant composées de $44 \%$ de méthane $\left[\mathrm{CH}_{4}\right], 29 \%$ de protoxyde d'azote $\left(\mathrm{N}_{2} \mathrm{O}\right)$ et $27 \%$ de dioxyde de carbone $\left(\mathrm{CO}_{2}\right]$. Ces chiffres globaux recouvrent toutefois des réalités très différentes de productivité et d'émission en fonction des régions et des types d'élevage. Le débat actuel sur l'agriculture et le climat y pointe l'élevage pastoral au Sahel : la faible productivité des animaux et l'ingestion de fourrages grossiers, qui composent l'essentiel de leur alimentation, induisent une forte émission de méthane par unité de produit animal (lait ou viande). Mais l'impact de cet élevage sur le changement climatique n'a jamais été évalué précisément parce que les écosystèmes pastoraux des régions sèches sont complexes et peu étudiés sous cet angle.

L'élevage pastoral sahélien joue un rôle primordial dans l'exploitation de ce milieu très contraint (voir encadré p. 3). Quelle est sa réelle contribution au bilan carbone des territoires ? L'estimation de ce bilan est complexe : il faut s'intéresser à tous les éléments de l'écosystème et à leurs interactions, ce qui inclut les animaux, des saisons et des lieux investis par les troupeaux. Ces résultats incitent à revoir à la baisse les normes actuelles de calcul du comportement alimentaire et des émissions de méthane issu de la digestion des ruminants. D'autres implications sont envisageables, comme améliorer le bilan carbone par des pratiques locales spécifiques et valoriser ces espaces sur le marché du carbone. Préserver ce mode d'élevage est aussi une des voies pour générer du développement et contribuer à sécuriser ces régions.

leurs déplacements et leur accès à l'eau, les plantes et les sols. Une recherche récente au Sénégal a contribué à mieux aborder cette problématique, avec des implications pour les politiques publiques de ces régions.

\section{Résultats clés au Sahel : émission de méthane} des ruminants et variation du bilan carbone

L'étude, menée au nord du Sénégal (voir encadré p. 2), a mis à jour de nouvelles connaissances sur ces écosystèmes. Elle a utilisé une méthode originale pour estimer le comportement alimentaire des ruminants et l'émission de méthane issu de leur digestion, appelé « méthane entérique ».

Sur l'année complète, les troupeaux (bovins, ovins, caprins] mangent moins d'un tiers de la biomasse herbacée disponible et moins de $5 \%$ des feuilles des arbres et arbustes du territoire pastoral. Les mesures de l'ingestion quotidienne montrent que la norme moyenne utilisée en Afrique doit être remise en question : à $25 \mathrm{~g} / \mathrm{kg}$ (grammes de matière sèche végétale par kilogramme de poids vif d'animal), cette norme est trop élevée pour les bovins et trop basse pour les petits ruminants. L'étude propose de nouvelles normes basées sur les mesures quotidiennes réalisées

$>$ ingestion de $18 \mathrm{~g} / \mathrm{kg}$ pour les bovins et de $34 \mathrm{~g} / \mathrm{kg}$ pour les petits ruminants

$>$ ou une norme applicable à tous types de ruminants, de 73 grammes par kilogramme de poids métabolique - en nutrition, le poids métabolique correspond au poids vif à la puissance 0,75 et les besoins d'entretien d'un animal sont toujours proportionnels au poids métabolique. 
Modèle simplifié des émissions de gaz à effet de serre

et du stockage du carbone d'un territoire pastoral sahélien :

l'approche écosystémique du bilan carbone se base sur ce modèle.

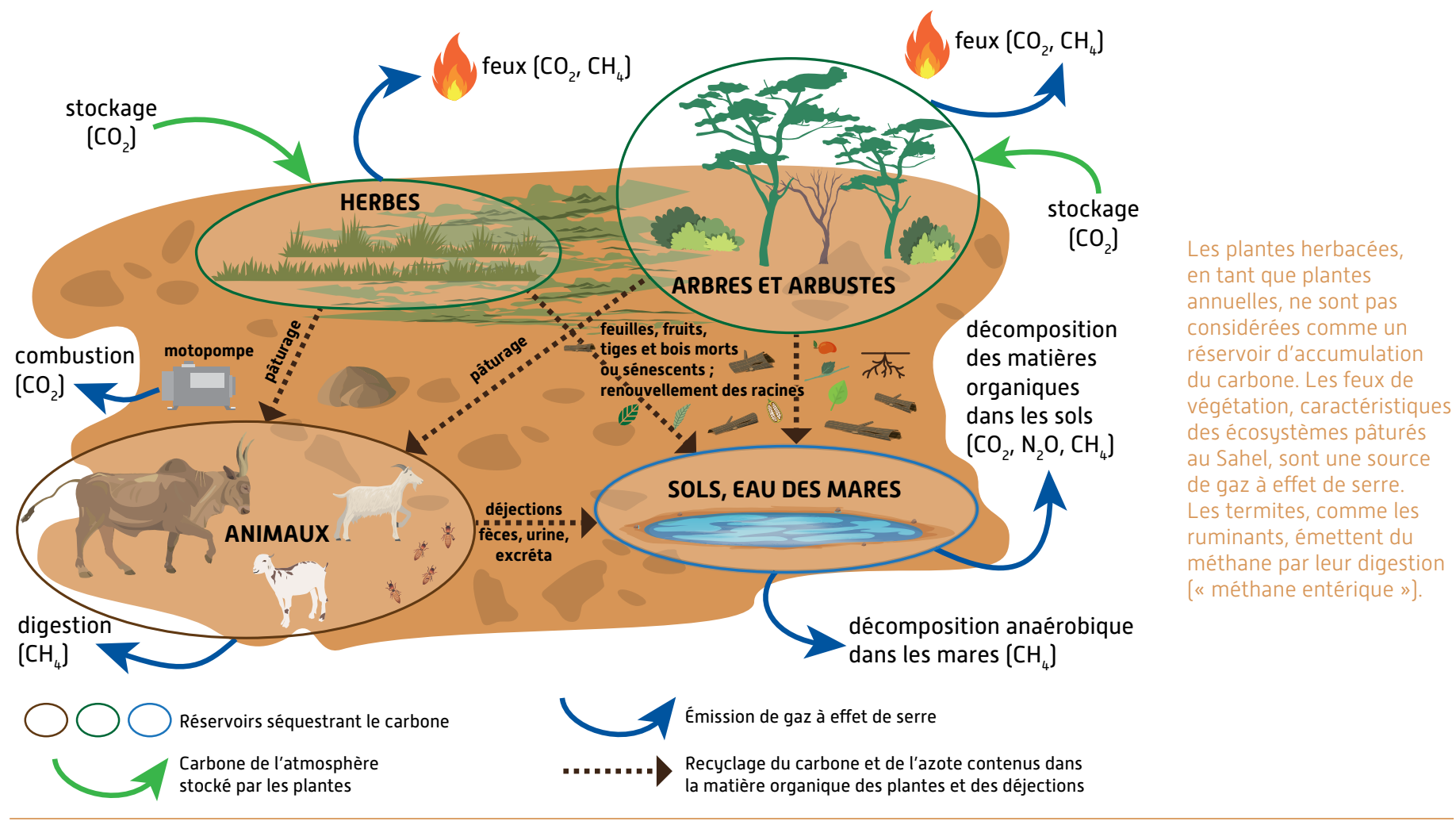

Le système pastoral sahélien est en équilibre :

qui émet les gaz à effet de serre et qui stocke le carbone ?

Le sol est le plus gros émetteur

(en pourcentage des émissions totales annuelles).

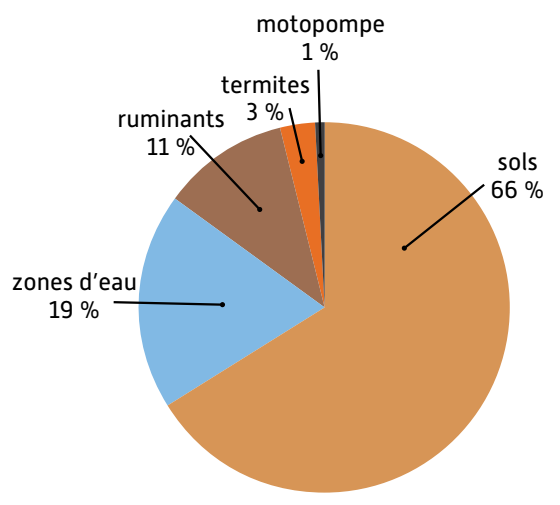

Les arbres et arbustes sont les plus gros stockeurs

(en pourcentage du stockage total annuel).

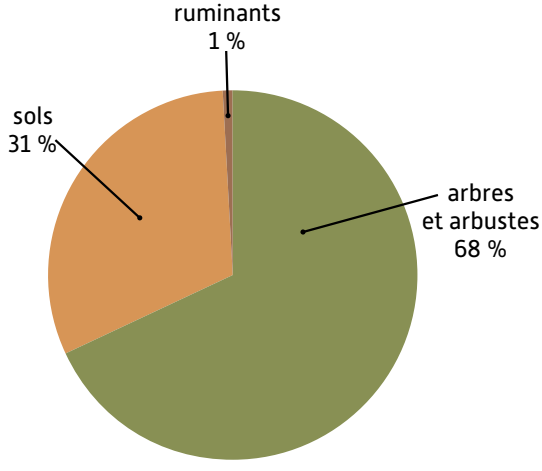

Pour l'année suivie, il n'y a pas eu de feux de brousse.
Un bilan carbone intégrant l'ensemble des éléments de l'écosystème : application au Sahel

Le bilan carbone d'un système est calculé en évaluant l'ensemble des émissions de gaz à effet de serre $\left(\mathrm{CH}_{4}, \mathrm{CO}_{2}, \mathrm{~N}_{2} \mathrm{O}\right]$ et le carbone qui est séquestré. Le bilan ramène l'ensemble de ces flux en équivalent carbone.

L'étude a été menée dans la région sylvopastorale du Ferlo au nord du Sénégal, plus précisément dans l'aire de desserte du forage de Widou $\left[706 \mathrm{~km}^{2}\right]$. Cette région au climat semi-aride est représentative des écosystèmes pastoraux sahéliens, où l'élevage pastoral est l'activité majeure. Pendant une année complète, le suivi mensuel de ce territoire a abouti à un bilan carbone à large échelle [voir figures].

La méthode écosystémique utilisée ici, dite aussi " territoriale ", prend en compte l'ensemble du fonctionnement écologique d'un territoire pour calculer son bilan carbone. Tous les échanges sont comptés, c'est-à-dire les émissions de gaz à effet de serre dans l'atmosphère et la séquestration du carbone dans l'écosystème. Le périmètre du territoire est défıni, puis ses principaux éléments [animaux, sols, plantes] et les interactions entre eux et avec I'atmosphère sont décrits. La méthode aboutit à une représentation spatiale et dynamique des émissions et des variations des stocks, en fonction des saisons et des lieux.

Cette méthode est bien adaptée au Sahel parce qu'il n'y a quasiment pas d'intrants extérieurs. Classiquement, les bilans se font à l'échelle d'une ferme en se focalisant sur les intrants sans tenir compte de la variabilité en cours d'année ni de l'hétérogénéité spatiale des processus écologiques à l'origine des émissions et de la séquestration. Au contraire, l'approche écosystémique prend en compte des processus spécifiques : par exemple, la présence d'animaux consommant les fourrages sur pied réduit le risque de feux et les termites. 
Ces nouvelles normes d'ingestion journalière sont modulables ( $\pm 12 \%$ ) selon les ressources du territoire, les pratiques d'élevage et les saisons. Malgré cela, elles ont des conséquences sur l'estimation des émissions de méthane entérique : pour les bovins sahéliens, les estimations officielles actuelles seraient le double de la réalité. Ces normes pourraient contribuer à réviser celles utilisées dans les lignes directrices du Groupe d'experts intergouvernemental sur l'évolution du climat (Giec).

Les travaux ont montré que le bilan carbone du territoire est à l'équilibre, même s'il varie selon le lieu et la saison. Dans la zone étudiée, un hectare d'écosystème pastoral émet pendant une année 0,71 tonne d'équivalent carbone et en séquestre 0,75 tonne: autrement dit, il stocke la différence, soit $40 \pm 6$ kilogrammes d'équivalent carbone. Au Sahel, le stockage dans les arbres, arbustes et sols compense les émissions de gaz à effet de serre des animaux liées à leur alimentation et au dépôt de leurs déjections (voir figures p. 2).

Plus finement, le bilan carbone annuel varie d'un lieu à l'autre et cette variation est liée aux pratiques d'élevage. Les parcours herbeux, arbustifs et arborés, où les animaux se déplacent pour brouter, sont des lieux où la séquestration prévaut. A l'inverse, les aires de repos proches des campements et les abords des points d'eau, qui reçoivent beaucoup de déjections, sont des lieux surtout émetteurs.

La variation saisonnière du bilan carbone, peu connue, a pu être mesurée pour l'ensemble de l'écosystème pastoral, tous lieux confondus. En saison des pluies, l'écosystème émet beaucoup plus de gaz à effet de serre qu'il ne stocke de carbone - les animaux et les points d'eau étant les principales sources d'émission. Inversement, en saison sèche, l'écosystème stocke - les déjections et les herbes étant enfouies dans le sol par le piétinement des animaux.

Même si l'étude a été menée lors d'une année à pluviosité inférieure à la normale, l'équilibre du bilan carbone des territoires pastoraux sahéliens n'est pas remis en cause, puisque la mobilité des troupeaux permet aux éleveurs de s'adapter à la production d'herbe et de réduire la pression sur les zones de pâturage (voir encadré ci-contre].

\section{Implications techniques et politiques: penser ces territoires comme un outil d'atténuation}

\section{du changement climatique}

Envisager localement trois options opérationnelles d'atténuation Le bilan carbone obtenu au nord du Sénégal apporte une image fine des variations d'émission et de stockage à la fois dans le temps et dans les différents lieux du territoire pastoral. Outre l'option reconnue de plantation arborée, telle que la Grande muraille verte en Afrique, trois techniques sont bien adaptées au pastoralisme: aménager les points d'eau, valoriser les déjections animales par la méthanisation, stocker du fourrage quand il est abondant et de qualité.

Les abords des points d'eau émettent un cinquième des gaz à effet de serre. Pour limiter cela, il faut y réduire le dépôt des déjections, en créant des abreuvoirs bien entretenus et en mettant en défend les zones où l'eau s'accumule en surface.

Les aires de parcage concentrent beaucoup de déjections. Des unités de méthanisation en valoriseraient la collecte pour produire du biogaz domestique et un sous-produit fertilisant recyclable en agriculture et en foresterie.

En une année, moins d'un tiers de l'herbe produite par l'écosystème est ingérée par les animaux, le reste étant restitué au sol par leur piétinement. Récolter une partie de l'herbe non broutée puis la stocker sous forme de foin suffirait aux besoins des troupeaux et permettrait un départ plus tardif en transhumance. L'herbe pourrait être coupée avant la fin de saison des pluies pour garantir une
La mobilité des troupeaux :

réponse à la variabilité du climat au Sahel

Le pastoralisme privilégie la mobilité des troupeaux, fondée sur l'accès partagé et la gestion communautaire des points d'eau (puits, forages, mares) et des parcours - espaces naturels herbeux et arborés, jachères et champs après récolte. La mobilité est une stratégie d'adaptation à la variabilité locale saisonnière et interannuelle des pluies et des ressources, ces dernières évoluant en fonction du climat.

Au Sahel, la végétation se régénère pendant les quatre mois de saison des pluies, de juillet à octobre. Ce stock renouvelé est aussi la ressource alimentaire principale des ruminants pendant les huit mois de saison sèche. L'éleveur mène son troupeau vers les meilleures ressources du moment, dans la limite d'un effort acceptable, ce qui l'oblige à se déplacer chaque jour et selon les saisons. En saison des pluies, les animaux se déplacent chaque jour autour du campement. En saison sèche, s'ajoutent les longs déplacements sur des dizaines ou centaines de kilomètres : c'est la transhumance, qui peut traverser plusieurs régions d'un pays et même passer d'un pays à l'autre.

Les éleveurs décident des itinéraires pour assurer l'abreuvement et, par le choix des plantes à brouter, les besoins alimentaires des animaux. Les ruminants sahéliens ont un potentiel génétique adapté aux pratiques pastorales, aux conditions extrêmes de chaleur et de sécheresse, et ils s'accommodent d'une large gamme de plantes.

Au cours de la saison sèche, dès que la qualité et la quantité de fourrage diminuent, les transhumances s'organisent, laissant à la résidence principale quelques animaux. Au fil de la transhumance, les animaux maigrissent, parce que l'herbe sèche est moins riche et que les déplacements demandent de l'énergie.

qualité optimale et assurer une repousse. Des banques fourragères pourraient être créées par les acteurs du territoire, avec tout un secteur à organiser - gérer les coupes, les lieux et les structures de stockage, la redistribution du stock et sa rémunération. Cela impliquerait un changement pour les éleveurs, qui n'ont pas l'habitude de collecter du fourrage, même si les sécheresses récentes et répétées ont poussé certains d'entre eux à en stocker. Cette innovation a un triple effet positif d'atténuation : risque de feux réduit, moins de méthane entérique du fait d'un meilleur fourrage, performances animales (viande et lait) augmentées. Ces pratiques favorisent aussi l'adaptation au changement climatique, car elles permettent de compenser en partie la variabilité de production végétale liée au climat, de réduire la vulnérabilité des systèmes pastoraux et d'améliorer les revenus des éleveurs.

Continuer la recherche dans d'autres écosystèmes à bas niveau d'intrants - La méthode écosystémique du bilan carbone demande à être répliquée plusieurs années dans d'autres territoires agricoles tropicaux, qu'il s'agisse de systèmes exclusivement à l'herbe ou de systèmes mixtes agriculture-élevage. Cela aidera à mieux évaluer les effets directs et indirects, négatifs ou positifs, des activités d'élevage sur le changement climatique. La multiplication de nouvelles références chiffrées d'émission de gaz à effet de serre et de séquestration de carbone réduirait les incertitudes et ces résultats pourraient être intégrés aux lignes directrices du Giec.

Valoriser les espaces pastoraux sur le marché international du carbone - En Afrique, ils s'étendent sur près de 1,3 million d'hectares, ce qui représente un potentiel significatif de séquestration. Les fonds verts et les crédits carbone pourraient aussi être mobilisés au profit de l'élevage pastoral dans les pays subsahariens. Le bilan carbone par hectare pourrait devenir un indicateur supplémentaire pour évaluer la performance d'un système d'élevage extensif à l'herbe dans les régions tropicales. Pour faire avancer le débat autour de l'élevage pastoral, ce nouvel indicateur s'inscrit 
parfaitement dans le processus de l'Action commune de Koronivia pour l'agriculture [Koronivia Joint Work on Agriculture) adoptée à la $23^{e}$ Conférence des parties de la convention-cadre des Nations Unies sur les changements climatiques (COP 23, 2017).

Renforcer les politiques de soutien à l'élevage pastoral - Dans les pays du Sahel, la problématique élevage et changement climatique nécessite de renforcer les connaissances et l'expertise sur ce sujet. Le potentiel d'atténuation des territoires pastoraux mis à jour par l'étude au Sénégal est un argument pour y remédier. Plus largement, en
Afrique subsaharienne, favoriser le maintien de la mobilité pastorale est une action clé pour préserver à la fois les populations et l'équilibre des écosystèmes. Cette mobilité est régulièrement menacée par l'insécurité et les conflits, la croissance démographique et l'expansion urbaine et agricole. Au-delà de son rôle atténuateur du changement climatique, l'élevage pastoral est une forme optimale d'occupation et de valorisation de vastes espaces peu propices aux autres activités c'est un des moyens pour sécuriser ces régions et y stabiliser les populations humaines.

Ce Perspective $\mathrm{n}^{\circ} 52$ est issu de travaux conduits au sein

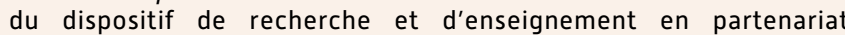
du Cirad Pôle Pastoralisme et Zones Sèches en Afrique de l'Ouest [dP PPZS, https://www.ppzs.org/] impliquant notamment le Cirad et I'Institut sénégalais de recherches agricoles (Isra, https://www.isra.sn/]. Il s'appuie aussi sur les résultats de recherche du projet européen ANIMALCHANGE (An Integration of Mitigation and Adaptation Options for Sustainable Livestock Production under Climate Change, 20112015, FP7-KBBE-2010 Grant agreement 266018, http://animalchange.eu/] Ces travaux ont notamment donné lieu aux publications suivantes

$>$ Assouma M. H., 2016. Approche écosystémique du bilan des gaz à effet de serre d'un territoire sylvo-pastoral sahélien : contribution de l'élevage. Paris, Montpellier, AgroParisTech, $230 \mathrm{p}$. Thèse de doctorat.

http://agritrop.cirad.fr/593394/

$>$ Assouma M. H., Hiernaux P., Lecomte P., Ickowicz A., Bernoux M., Vayssières J., 2019. Contrasted seasonal balances in a Sahelian pastoral ecosystem result in a neutral annual carbon balance. Journal of Arid Environments 162: 62-73. https://doi.org/10.1016/j.jaridenv.2018.11.013

> Assouma M. H., Lecomte P., Hiernaux P., Ickowicz A., Corniaux C., Decruyenaere V., Diarra A.R., Vayssières J., 2018. How to better account

\section{Quelques mots sur..}

Mohamed Habibou Assouma est agronome zootechnicien au Cirad à l'Umr Selmet (Systèmes d'élevage méditerranéens et tropicaux https://umr-selmet.cirad.fr/). II a conduit les travaux sur lesquels s'appuie ce numéro de Perspective dans le cadre de son doctorat de 2013 à 2016.

habibou.assouma@cirad.fr

Philippe Lecomte est agronome zootechnicien au Cirad à I'Umr Selmet à Montpellier. II a coordonné les activités du Cirad dans le cadre du projet européen ANIMALCHANGE [2011-2015, http://animalchange.eu/].

philippe.lecomte@cirad.fr

Christian Corniaux est agronome zootechnicien au Cirad à I'Umr Selmet Au Sénégal, il co-anime le dispositif de recherche et d'enseignement en partenariat du Cirad Pôle Pastoralisme et Zones Sèches en Afrique de l'Ouest [dP PPZS, https://www.ppzs.org/]. christian.corniaux@cirad.fr

Pierre Hiernaux est agronome écologue, expert des pâturages et des systèmes d'élevage extensifs en Afrique subsaharienne. Actuellement à la retraite, il est le responsable du bureau d'études français Pastoralisme Conseil [Pastoc].

pierre.hiernaux2@orange.fr

Alexandre Ickowicz est vétérinaire zootechnicien au Cirad et il dirige l'Umr Selmet. Il étudie le rôle des systèmes pastoraux dans le développement des territoires. Il est membre du Comité scientifıque français de la désertification [Csfd, http://www.csf-desertification.org/] et il co-anime le réseau d'action Restoring Value to Grasslands de la plateforme Global Agenda for Sustainable Livestock [http://www.livestockdialogue.org/en/].

$$
\text { alexandre.ickowicz@cirad.fr }
$$

Jonathan Vayssières est agronome modélisateur au Cirad à l'Umr Selmet. II a encadré les travaux de thèse ayant conduit à ces résultats lors de son affectation au Sénégal de 2011 à 2017. Il étudie le rôle de l'élevage dans I'organisation des cycles des nutriments et du carbone à différentes échelles (exploitation, filière, territoire) et dans divers agroécosystèmes.

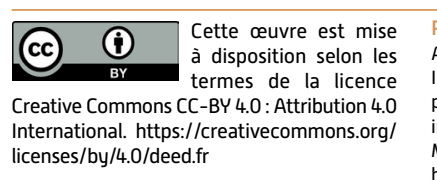
licenses/by/4.0/deed.f
Pour citer ce document

Assouma M. H., Lecomte P., Corniaux C., Hiernaux P., Ickowicz A., Vayssières J., 2019. Territoires d'ëlevage pastoral au Sahel : un bilan carbone avec un potentie

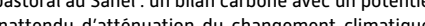
Montpellier, Cirad, Perspective 52. hontpellier, Cirad, Perspective 52 . for livestock diversity and fodder seasonality in assessing the fodder intake of livestock grazing semi-arid sub-Saharan Africa rangelands. Livestock Science 216: 16-23. https://doi.org/10.1016/j.livsci.2018.07.002 > Assouma M. H., Serça D., Guérin F., Blanfort V., Lecomte P., Touré I., Ickowicz A., Manlay R.J., Bernoux M., Vayssières J., 2017. Livestock induces strong spatial heterogeneity of soil $\mathrm{CO}_{2}, \mathrm{~N}_{2} \mathrm{O}, \mathrm{CH}_{4}$ emissions within a semi-arid sylvo-pastoral landscape in West Africa. Journal of Arid Land 9: 210-221. https://doi.org/10.1007/s40333-017-0001-y

> Rasmussen K., Brandt M., Tong X., Hiernaux P., Diouf A.A., Assouma M. H., Tucker C.J., Fensholt R., 2018. Does grazing cause land degradation? Evidence from the sandy Ferlo in Northern Senegal. Land Degradation \& Development 29: 4337-4347. https://doi.org/10.1002/ldr.3170

> Vayssières J., Assouma M. H., Lecomte P., Hiernaux P., Bourgoin J., Jankowski F., Corniaux C., Vigne M., Torquebiau E., Ickowicz A., 2017. L'élevage au cœur de paysages " climato-intelligents " en Afrique de l'Ouest. In : Des territoires vivants pour transformer le monde, Caron P., Valette E., Wassenaar T., Coppens D'Eeckenbrugge G., Papazian V. [Éds]. Versailles, Éditions Quae, pp. 114-120. ISBN 978-2-7592-2654-2. http://agritrop.cirad.fr/583600/

\section{Quelques liens}

Agence panafricaine de la Grande muraille verte.

https://www.grandemurailleverte.org/

Gerber P.J., Steinfeld H., Henderson B., Mottet A., Opio C., Dijkman J., Falcucci A., Tempio G., 2013. Tackling climate change through livestock - A global assessment of emissions and mitigation opportunities. Fao, Rome, $139 \mathrm{p}$. ISBN 978-92-5-107920-1. http://www.fao.org/3/i3437e/i3437e00.htm

Groupe d'experts de haut niveau sur la sécurité alimentaire et la nutrition du Comité de la sécurité alimentaire mondiale (HLPE), 2016. Le développement agricole durable au service de la sécurité alimentaire et de la nutrition : quels rôles pour l'élevage? Rapport N¹0 du HLPE, Rome, 158 p.

http://www.fao.org/cfs/cfs-hlpe/rapports/report-10-elaboration-process/fr/ Groupe d'experts intergouvernemental sur l'évolution du climat (Giec, Intergovernmental Panel on Climate Change, IPCC). https://www.ipcc.ch/

IPCC, 2006. 2006 IPCC Guidelines for National Greenhouse Gas Inventories, Prepared by the National Greenhouse Gas Inventories Programme, Eggleston H.S., Buendia L., Miwa K., Ngara T. and Tanabe K. (eds). Institute for Global Environmental Strategies (IGES), IPCC, Hayama, Japan. ISBN 4-88788-032-4. https://www.ipcc-nggip.iges.or.jp/public/2006gl/

Mayaux P., Bartholomé E., Fritz S., Belward A., 2004. A new land-cover map of Africa for the year 2000. Journal of Biogeography 31: 861-877. https://doi.org/10.1111/j.1365-2699.2004.01073.x

Organisation des Nations Unies pour l'alimentation et I'agriculture (Fao) L'élevage et l'environnement. http://www.fao.org/livestock-environment/fr/

United Nations Climate Change, 2017. Issues related to agriculture. Decision 4/CP.23: "Koronivia joint work on agriculture".

https://unfccc.int/topics/land-use/workstreams/agriculture

\section{Directeur de la publication : Michel Eddi, C Président directeur général du Cirad LARECHERCHEAGRONOMIOUE Rédacteur.rice.s en chef : Patrick Caron LARECHERCHE AGRONOMIQUE Umr ART-Dev (Acteurs, ressources et
POURLEDÉVELOPPEMENT POUR LE DÉVELOPPEMENT territoires dans le développement] 42, rue Scheffer 75116 Paris • France www.cirad.fr \\ scientifique et technique \\ Étienne Hainzelin, direction générale \\ Mise en pages et illustrations : Laurence Laffont \\ Diffusion : Christiane Mastri, délégation \\ à la communication \\ www.cirad.fr/publications-ressources/edition/perspective-policy-brief perspactive ISSN-L 2275-9131 - Email : perspective@cirad.fr}

\section{a. M U S E}

MONTPELLER UNIVERSTIÉD'EXCELLENCE https://muse.edu.umontpellier.fr 